\title{
Stability of Milling Process with Variable Spindle Speed Using Runge-Kutta-Based Complete Method
}

\author{
Shujie Lv $\mathbb{D}^{1}$ and Yang Zhao ${ }^{2}$ \\ ${ }^{1}$ Technology Department, Siyu (Shanghai) Co Ltd, Shanghai 201112, China \\ ${ }^{2}$ State Key Laboratory for Strength and Vibration of Mechanical Structures, Xi'an Jiaotong University, Xi'an 710049, China
}

Correspondence should be addressed to Shujie Lv; 141860055@st.usst.edu.cn

Received 8 November 2020; Revised 26 December 2020; Accepted 16 January 2021; Published 10 February 2021

Academic Editor: Jerzy Baranowski

Copyright (c) 2021 Shujie Lv and Yang Zhao. This is an open access article distributed under the Creative Commons Attribution License, which permits unrestricted use, distribution, and reproduction in any medium, provided the original work is properly cited.

\begin{abstract}
The variable-spindle-speed (VSS) technique is effective in preventing regenerative chatter in milling processes. However, spindlespeed-modulation parameters should be deliberately selected to augment the material removal rate. Stability-prediction algorithms of stability predicting play an important role in this respect, as they allow the prediction of stability for all ranges of a given spindle speed. The increase in calculation time in variable-spindle-speed milling, which is caused by the modulation frequency, hinders its practical use in the workshop. In this paper, a Runge-Kutta-based complete discretization method (RKCDM) is presented to predict the stability of milling with variable spindle speeds, which is described by a set of delay differential equations (DDEs) with time-periodic coefficients and time-varying delay. The convergence and calculation efficiency are compared with those of the semidiscretization method (SDM) under different testing configurations and milling conditions. Results show that RKCDM is more accurate and saves at least $50 \%$ of the calculation time of SDM. The effects of modulation parameters on the stability of VSS milling are explored through stability lobe diagrams produced from RKCDM.
\end{abstract}

\section{Introduction}

In the milling process, chatter caused by vibrations between the cutter and workpiece hinders manufacturing production. It cannot only reduce machining performance, but can cause a poor workpiece-surface finish, increase tool wear, and decrease the lifetime of the machine tool itself.

Chatter is generally classified in two categories. Primary chatter is usually triggered by friction between the cutter and the workpiece. Secondary chatter, which is caused by a regenerative mechanism, is most common factor. That is why many publications discuss only regenerative chatter.

Several strategies have been proposed to suppress or avoid chatter. They can be roughly categorized as follows:

(1) Strategies based on a stability lobe diagram (SLD) and the selection of cutting parameters

(2) Active chatter-suppression techniques to decrease the vibration of a machine tool system
(3) Semiactive chatter-suppression strategies

(4) Passive chatter-suppression strategies accounting for a machine tool's compliance or configuration

(5) Other strategies based on signal surveillance in the milling process for the adjustment of milling parameters

SLD is the most logical and widely accepted method to select cutting parameters to avoid chatter. Researchers have proposed numerous methods to predict machining stability. These include the zero-order frequency method [1], numerical integration method [2], temporal finite element analysis (TFEA) [3], semidiscretization method (SDM) $[4,5]$, semidiscretization method on a Shannon standard orthogonal basis [6, 7], Lambert $\mathrm{W}$ function-based method [8], Legendre polynomial-based method [9], Adams-Simpson-based method [10], Runge-Kutta-based complete discretization method [11], and Chebyshev collocation method for delay differential equations [12]. 
Because regenerative chatter is a result of improper phase differences between undulate surfaces caused by successive cuts by a tool, alternative methods usually vary the time lag between cutter teeth. Slavicek [13] demonstrated that this can be achieved through variable-pitch cutters. Altintas et al. [14], Olgac and Sipahi [15], Jin et al. [16], and Sims et al. [17] all demonstrated that variable-pitch cutters can avoid or significantly suppress chatter.

The idea of changing the spindle speed to suppress or avoid chatter in machining dates back to the 1970s and the pioneering research of Stoferle and Grab [18]. After that, Takemura et al. [19] studied the stability of variable-spindlespeed turning, and experimental tests showed only small improvements in the depth of cuts, although the stability of VSS was higher than that of constant-spindle-speed (CSS) milling. Altintas and Chan [20] investigated the stability of VSS with sinusoidal modulation using a time-simulation method. Tsao et al. [21] described the VSS milling system in the angle domain and showed that chatter could be suppressed or eliminated to a great extent by properly selecting the modulation parameters. Long and Balachandran [22] analyzed the stability of up- and down-milling with sinusoidal modulation using the semidiscretization method. In the frequency domain, Zatarain et al. [23] proposed a general theory to analyze the stability of VSS milling. Seguy et al. $[24,25]$ used a semidiscretization method to study the stability of VSS milling in the high-speed domain and reported that VSS was an effective method to reduce perioddoubling chatter. Based on the full-discretization method, Xie and Zhang [26, 27] presented an improved SDM to predict the stability of VSS milling. Ding et al. [28] studied the stability of the VSS milling system using the constantstep numerical method. Niu et al. [29] unified different periodic time-variant spindle-speed-modulation schemes in a unique framework, derived and calculated the timevarying delay based on the fourth-order Runge-Kutta method, and calculated and compared the stability of VSS milling with different modulation schemes using variablestep numerical integration. Totis et al. [30] studied the stability of milling with VSS in the angular domain. Jin et al. [31] studied the stability of milling with constant pitch, variable pitch, and VSS, using a full-discretization method.

As mentioned above, much research has been done to predict the stability of VSS milling. However, the required computational time increases greatly due to frequency modulation, and this hinders the application of VSS [29]. Niu et al. [32] and Li et al. [11] recently proposed a Runge-Kutta-based complete discretization method (RKCDM) using complete discretization scheme [33], but their research was based exclusively on CSS machining. In this paper, we present a two-degree-of-freedom dynamic milling model expressed by DDEs and a Runge-Kutta-based complete discretization method for efficient analysis of VSS milling. The convergence and computational efficiency are compared with a state-of-the-art semidiscretization method. Finally, we discuss the VSS modulation effects on the stability of milling with the aid of maps and contour plots of axial depth of cut (ADOC) calculated by RKCDM.

\section{Modeling of VSS Process}

2.1. VSS. In this paper, periodic spindle-speed variation with sinusoidal modulation will be considered and analyzed. As shown in Figure 1, a sinusoidal spindle-speed variation $\Omega(t)$ is periodic at a time period $T=60 / \Omega_{0} / \mathrm{RVF}$, with a nominal value $\Omega_{0}$, and an amplitude of the speed variation $\Omega_{A}=\mathrm{RVA} \times \Omega_{0}$. The shape function of the sinusoidal modulation is modeled as

$$
\begin{aligned}
\Omega(t) & =\Omega_{0}+\Omega_{A} \sin \left(\frac{2 \pi}{T} t+\eta\right) \\
& =\Omega_{0}\left\lceil 1+\mathrm{RVA} \cdot \sin \left(\operatorname{RVF} \frac{2 \pi}{60} \Omega_{0} t+\eta\right)\right\rceil,
\end{aligned}
$$

where $\mathrm{RVA}=\Omega_{A} / \Omega_{0}$ is the ratio of the speed-variation amplitude to the nominal spindle speed, $\operatorname{RVF}=60 /\left(\Omega_{0} T\right)$ is the ratio of the speed-variation frequency to the nominal spindle speed, and $\eta$ is the phase angle of the sinusoidal modulation at time $t=0$.

2.2. Structural Model. In the machining process, relatively more flexible parts will undergo dynamic deflection and generate an undulating surface. So, the system can be simplified to an oscillator motivated by the force of milling. We show an example model [34] in Figure 2. The governing equation of the model is

$$
\begin{aligned}
& {\left[\begin{array}{l}
x(t) \\
y(t)
\end{array}\right]+\left[\begin{array}{cc}
2 \zeta \omega_{n} & 0 \\
0 & 2 \zeta \omega n
\end{array}\right]\left[\begin{array}{l}
x(t) \\
y(t)
\end{array}\right]} \\
& +\left[\begin{array}{cc}
\omega_{n}^{2}+\frac{w h_{x x}(t)}{m_{t}} & \frac{w h_{x y}(t)}{m_{t}} \\
\frac{w h_{y x}(t)}{m_{t}} & \omega_{n}^{2}+\frac{w h_{x x}(t)}{m_{t}}
\end{array}\right]\left[\begin{array}{l}
x(t) \\
y(t)
\end{array}\right] \\
& =\left[\begin{array}{l}
\frac{w h_{x x}(t)}{m_{t}} \frac{w h_{x y}(t)}{m_{t}} \\
\left.\frac{w h_{y x}(t)}{m_{t}} \frac{w h_{y y}(t)}{m_{t}}\right]\left[\begin{array}{l}
x(t-\tau(t)) \\
y(t-\tau(t))
\end{array}\right],
\end{array}\right.
\end{aligned}
$$

where $\zeta$ is the relative damping, $\omega_{n}$ is the angular natural frequency, $w$ is the axial depth of cut, and $m_{t}$ is the modal mass of the teeth. The dynamic characteristic is assumed to be identical in the $x$ and $y$ directions, so the model mass $m_{t}$, the relative damping $\zeta$, and the natural frequency $\omega_{n}$ are the same in both directions. The time-varying cutting force coefficients, $h_{x x}(t), h_{x y}(t), h_{y x}(t)$, and $h_{y y}(t)$, are expressed as 


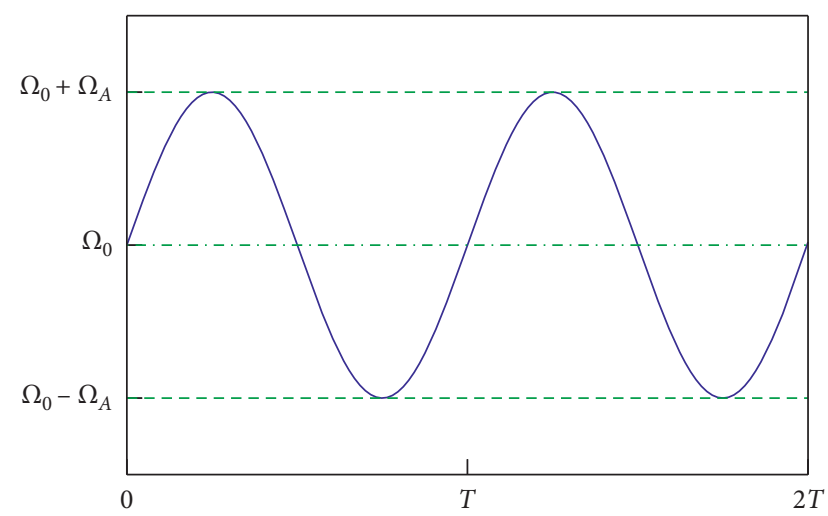

Figure 1: The sinusoidal modulation of the spindle speed.

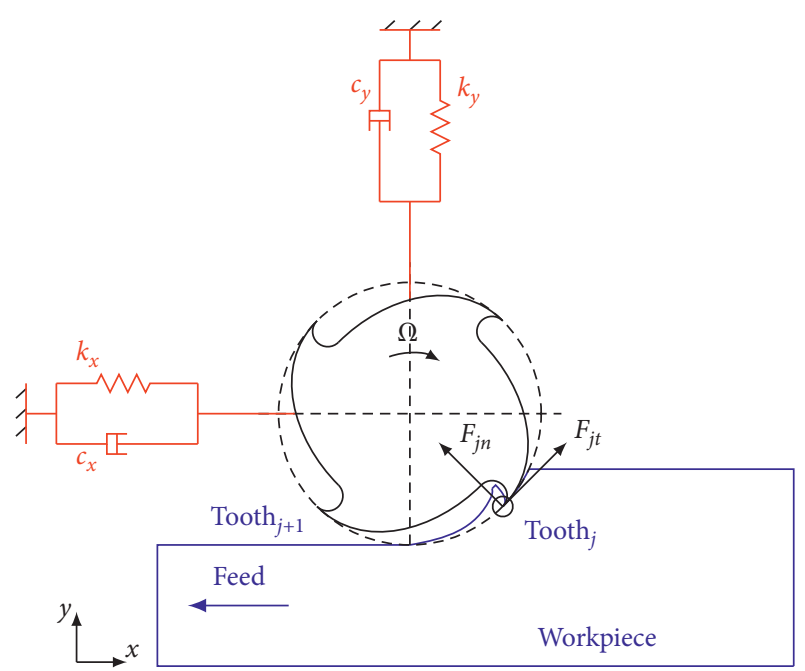

FIGURE 2: Schematic drawing of the end milling system.

$$
\begin{aligned}
& h_{x x}(t)=\sum_{j=1}^{N} g\left(\phi_{j}(t)\right) \sin \left(\phi_{j}(t)\right)\left(K_{t} \cos \left(\phi_{j}(t)\right)+K_{n} \cos \left(\phi_{j}(t)\right)\right), \\
& h_{x y}(t)=\sum_{j=1}^{N} g\left(\phi_{j}(t)\right) \cos \left(\phi_{j}(t)\right)\left(K_{t} \cos \left(\phi_{j}(t)\right)+K_{n} \sin \left(\phi_{j}(t)\right)\right), \\
& h_{y x}(t)=\sum_{j=1}^{N} g\left(\phi_{j}(t)\right) \sin \left(\phi_{j}(t)\right)\left(-K_{t} \sin \left(\phi_{j}(t)\right)+K_{n} \cos \left(\phi_{j}(t)\right)\right), \\
& h_{y y}(t)=\sum_{j=1}^{N} g\left(\phi_{j}(t)\right) \cos \left(\phi_{j}(t)\right)\left(-K_{t} \sin \left(\phi_{j}(t)\right)+K_{n} \cos \left(\phi_{j}(t)\right)\right),
\end{aligned}
$$

where $N$ is the number of cutter teeth, and $K_{t}$ and $K_{n}$ are, respectively, the tangential and normal linearized cuttingforce coefficients. The $j$-th tooth angular position $\phi_{j}(t)$ is defined as

$$
\phi_{j}(t)=\frac{2 \pi}{60} \int_{0}^{t} \Omega(s) \mathrm{d} s+(j-1) \frac{2 \pi}{N} .
$$

The window function $g\left(\phi_{j}(t)\right)$ can be defined as

$$
g\left(\phi_{j}(t)\right)= \begin{cases}1, & \text { if } \phi_{\mathrm{st}}<\phi_{j}(t)<\phi_{\mathrm{ex}} \\ 0, & \text { otherwise }\end{cases}
$$

where $\phi_{\text {st }}$ and $\phi_{\mathrm{ex}}$ are, respectively, the start and exit angles of the $j$-th cutter tooth. For up-milling, $\phi_{\text {st }}=0$ and $\phi_{\text {ex }}=\arccos (1-2 \cdot \mathrm{aD})$. For down-milling, $\phi_{\text {st }}=\arccos (1-$ $2 \cdot \mathrm{aD})$ and $\phi_{\mathrm{ex}}=0$, where $\mathrm{aD}=r / D$ is the radial immersion ratio, $r$ is the radial depth of cut, and $D$ is the cutter diameter.

2.3. Calculation of Variable Time Delays. Substituting (1) into (4), the angular position of the $j$-th tooth can be expressed as

$$
\phi_{j}(t)=\frac{2 \pi}{60}\left(\Omega_{0} t+\frac{60 \mathrm{RVA}}{2 \pi \mathrm{RVF}}\left(1-\cos \left(\omega_{m} t\right)\right)\right)+(j-1) \frac{2 \pi}{N},
$$

where $\omega_{m}=2 \pi / T$ is the angular velocity.

In this case, the time delay $\tau(t)$ cannot be given in an explicit form, as it is determined by an equation:

$$
\int_{t-\tau(t)}^{t} \frac{\Omega(s)}{60} \mathrm{~d} s=\frac{1}{N} .
$$

To calculate $\tau(t)$ directly is time-consuming [28], so we simplify the integral equation. By substituting (1) into (7), we achieve an implicit relationship:

$$
\frac{1}{60}\left(\Omega_{0} \tau(t)+\frac{\Omega_{1}}{\omega_{m}} \cos \left(\omega_{m}(t-\tau(t))\right)-\frac{\Omega_{1}}{\omega_{m}} \cos \left(\omega_{m} t\right)\right)=\frac{1}{N} .
$$

The function $\tau(t)$ cannot be solved in an explicit form; hence, it must be calculated numerically. However, if RVA and RVF are small enough; then, $\tau(t)$ is approximately [22]

$$
\tau(t) \approx \tau_{0}-\tau_{1} \sin \left(\omega_{m} t\right)=\tau_{0}\left(1-\mathrm{RVA} \cdot \sin \left(\mathrm{RVF} \cdot \frac{2 \pi}{60} \Omega_{0} t\right)\right),
$$

where $\tau_{0}=60 /\left(N \Omega_{0}\right)$ and $\tau_{1} / \tau_{0}=\Omega_{1} / \Omega_{0}$. As shown in Figure 3 , the exact time delays which is calculated using numerical solution from (8) and the approximate values from (9) is consistency. So, the approximation in (9) is effective. Figures 3(a) and 3(b) show that the approximated error for $\mathrm{RVA}=0.1$ is less than $\mathrm{RVF}=0.2$ because a small value of RVA is a condition of (9). Below, we will calculate time-varying delay through (9).

\section{Stability of Milling with VSS}

It must be pointed out that although VSS with sinusoidal modulation is discussed here, the proposed RKCDM can deal with other modulation types of VSS with no further difficulty. Through algebraic transformation, (2) can be written as

$$
\dot{\mathbf{x}}(t)=\mathbf{A}(t) \mathbf{x}(t)+\mathbf{B}(t) \mathbf{x}(t-\tau(t))
$$

where 


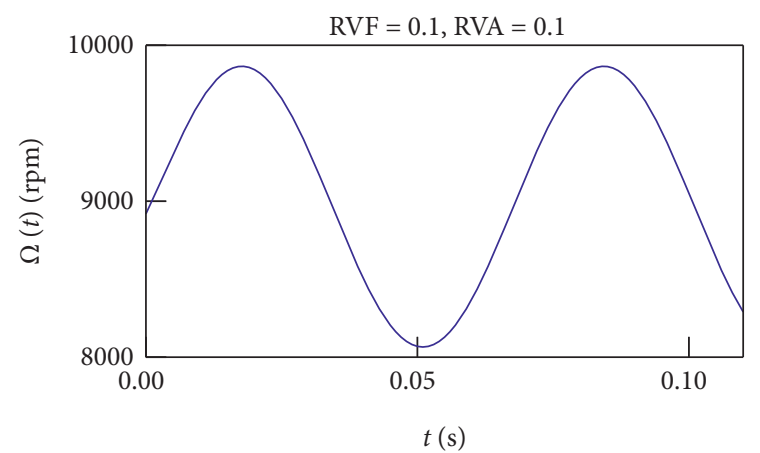

(a)

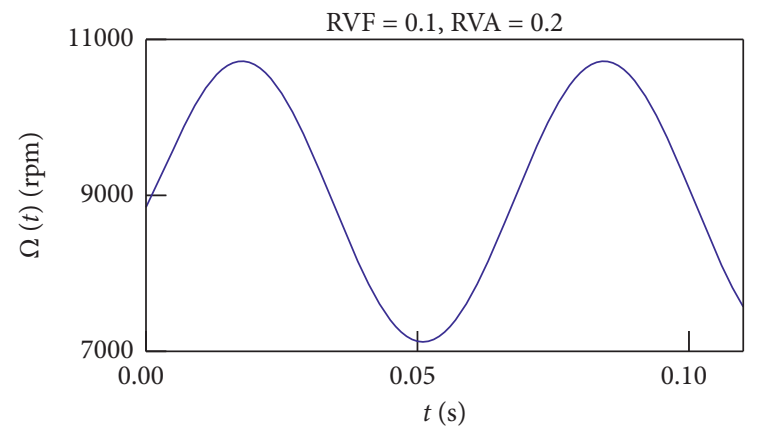

(c)

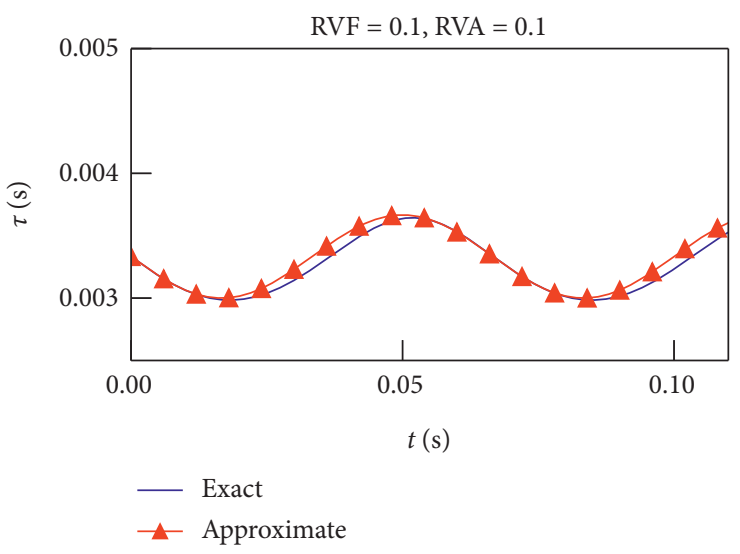

(b)

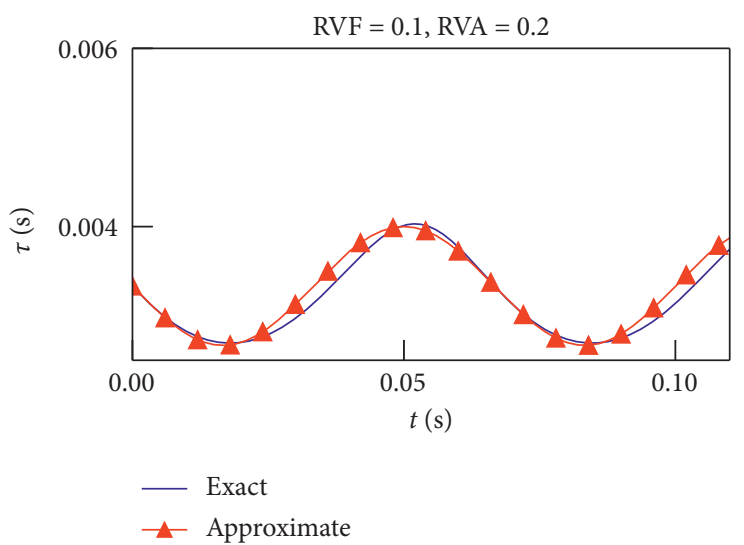

(d)

FIGURE 3: Comparison of corresponding exact and approximate time-delay variations for $\Omega_{0}=9,000 \mathrm{rpm}$ with sinusoidal spindle-speed variation.

$$
\begin{gathered}
\mathbf{A}(t)=\left[\begin{array}{cc}
0 & \mathbf{I} \\
-\mathbf{C}-\mathbf{H} & -\mathbf{K}
\end{array}\right], \mathbf{B}(t)=\left[\begin{array}{cc}
0 & 0 \\
-\mathbf{H} & 0
\end{array}\right], \mathbf{x}(t)=\left[\begin{array}{c}
x(t) \\
\dot{x}(t) \\
\dot{y}(t)
\end{array}\right], \\
\mathbf{C}=\left[\begin{array}{cc}
\omega_{n}^{2} & 0 \\
0 & \omega_{n}^{2}
\end{array}\right], \mathbf{K}=\left[\begin{array}{cc}
2 \zeta \omega_{n} & 0 \\
0 & 2 \zeta \omega_{n}
\end{array}\right], \mathbf{H}=\left[\begin{array}{c}
\frac{w h_{\mathrm{xx}}(t)}{m_{t}} \frac{w h_{\mathrm{xy}}(t)}{m_{t}} \\
\frac{w h_{\mathrm{yx}}(t)}{m_{t}} \frac{w h_{\mathrm{yy}}(t)}{m_{t}}
\end{array}\right] .
\end{gathered}
$$

The first step of discretization is construction of the timeinterval discretization of $\left\lfloor t_{i}, t_{i+1}\right\rfloor$ with length $\Delta t, i=0,1, \ldots$, such that $T=k \Delta t$, where $k$ is an integer that can be considered an approximation parameter referring to the time period. The time-step $\Delta t$ can be expressed as

$$
\Delta t=t_{i+1}-t_{i}=\frac{T}{k}=\frac{2 \pi}{k \omega_{m}}=\frac{60}{k \cdot \operatorname{RVF} \cdot \Omega_{0}} .
$$

The average delay for the discretization interval $\left\lfloor t_{i}, t_{i+1}\right\rfloor$ is defined as

$$
\tau_{i}=\frac{1}{\Delta t} \int_{t_{i}}^{t_{i+1}} \tau(t) \mathrm{d} t
$$

The series of integers $m_{i}$ is introduced as follows:

$$
m_{i}=\operatorname{int}\left(\frac{\tau_{i}+\Delta t / 2}{\Delta t}\right)=\operatorname{int}\left(\frac{k \cdot \mathrm{RVF} \cdot\left(1-\mathrm{RVA} c_{i}\right)}{N}+0.5\right)
$$

since

$$
\frac{\tau_{i}}{\Delta t}=\frac{\tau_{0}+\tau_{1} c_{i}}{\Delta t}=\frac{\tau_{0}}{\Delta t}\left(1-\mathrm{RVA}_{i}\right)=\frac{k \cdot \mathrm{RVF}}{N}\left(1-\mathrm{RVA}_{i}\right),
$$

and

$$
c_{i}=\frac{k}{2 \pi} \int_{i 2 \pi / k}^{(i+1) 2 \pi / k} \sin (t) \mathrm{d} t, \quad i=0,1, \ldots, k-1,
$$

where int $\left({ }^{*}\right)$ is the function that rounds positive numbers toward zero (e.g., int $(7.875)=7)$. Since the delay varies periodically over time, integer $m_{i}$ might vary for different semidiscretization steps. 
The maximum value of $m_{i}$ is introduced as

$$
M=\max _{i=1,2, \ldots}\left\{m_{i}\right\} .
$$

Note that $M$ can be considered an approximation parameter regarding the length of the time delay. is [35]

The most popular version of the Runge-Kutta algorithm

$$
\begin{aligned}
& \mathbf{x}_{i+1}=x_{i}+\frac{1}{6}\left(\mathbf{K}_{0}+2 \mathbf{K}_{1}+2 \mathbf{K}_{2}+\mathbf{K}_{3}\right), \\
& \mathbf{K}_{0}=\Delta t f\left(t_{i}, \mathbf{x}_{i}\right), \\
& \mathbf{K}_{1}=\Delta t f\left(t_{i}+\frac{\Delta t}{2}, \mathbf{x}_{i}+\frac{1}{2} \mathbf{K}_{0}\right), \\
& \mathbf{K}_{2}=\Delta t f\left(t_{i}+\frac{\Delta t}{2}, \mathbf{x}_{i}+\frac{1}{2} \mathbf{K}_{1}\right), \\
& \mathbf{K}_{3}=\Delta t f\left(t_{i}+\Delta t, \mathbf{x}_{i}+\mathbf{K}_{2}\right),
\end{aligned}
$$

where $x_{i}$ denotes $x(i \Delta t), x_{t+1}$ denotes $x((i+1) \Delta t)$, and $t_{i}$ represents $i \Delta t, i \in Z$ and $0 \leq i \leq m_{i}$. The following equations can be deduced in sequence to solve (10):

$$
\begin{aligned}
& \mathbf{K}_{0}=\Delta t \mathbf{A}_{i} \mathbf{x}_{i}+\Delta t B_{i} \mathbf{x}_{i-m_{i}}, \\
& \mathbf{K}_{1}=\mathbf{F}_{i, 1} \mathbf{x}_{i}+\mathbf{F}_{i-m_{i}, 1} \mathbf{x}_{i-m_{i}}+\mathbf{F}_{i-m_{i}+1,1} \mathbf{x}_{i-m_{i}+1}, \\
& \mathbf{K}_{2}=\mathbf{F}_{i, 2} \mathbf{x}_{i}+\mathbf{F}_{i-m_{i}, 2} \mathbf{x}_{i-m_{i}}+\mathbf{F}_{i-m_{i}+1,2} \mathbf{x}_{i-m_{i}+1}, \\
& \mathbf{K}_{3}=\mathbf{F}_{i, 3} \mathbf{x}_{i}+\mathbf{F}_{i-m_{i}, 3} \mathbf{x}_{i-m_{i}}+\mathbf{F}_{i-m_{i}+1,3} \mathbf{x}_{i-m_{i}+1},
\end{aligned}
$$

where

$$
\begin{aligned}
\mathbf{A}(t) & =\mathbf{A}_{i}+\frac{1}{2}\left(\mathbf{A}_{i+1}-\mathbf{A}_{i}\right), \\
\mathbf{B}(t) & =\mathbf{B}_{i}+\frac{1}{2}\left(\mathbf{B}_{i+1}-\mathbf{B}_{i}\right), \\
\mathbf{F}_{i, 1} & =\Delta t \mathbf{A}_{i+1 / 2}+\frac{1}{2} \Delta t^{2} \mathbf{A}_{i+1 / 2} \mathbf{A}_{i}, \\
\mathbf{F}_{i-m_{i}, 1} & =\frac{1}{2} \Delta t^{2} \mathbf{A}_{i+1 / 2} \mathbf{B}_{i}+\Delta t \frac{1}{2} \mathbf{B}_{i+1 / 2}, \\
\mathbf{F}_{i-m_{i}+1,1} & =\frac{1}{2} \Delta t \mathbf{B}_{i+1 / 2}, \\
\mathbf{F}_{i, 2} & =\Delta t \mathbf{A}_{i+1 / 2}+\frac{1}{2} \Delta t^{2} \mathbf{A}_{i+1 / 2} F_{i, 1}, \\
\mathbf{F}_{i-m_{i}, 2} & =\frac{1}{2} \Delta t^{2} \mathbf{A}_{i+1 / 2} \mathbf{F}_{i-m_{i}, 1}+\Delta t \frac{1}{2} \mathbf{B}_{i+1 / 2}, \\
\mathbf{F}_{i-m_{i}}+1,2 & =\frac{1}{2} \Delta t^{2} \mathbf{A}_{i+1 / 2} \mathbf{F}_{i-m_{i}+1,1}+\Delta t \frac{1}{2} \mathbf{B}_{i+1 / 2}, \\
\mathbf{F}_{i, 3} & =\Delta t \mathbf{A}_{i+1}+\Delta t^{2} \mathbf{A}_{i+1} \mathbf{F}_{i, 2}, \\
\mathbf{F}_{i-m_{i}, 3} & =\Delta t^{2} \mathbf{A}_{i+1} \mathbf{F}_{i-m_{i}, 2}, \\
\mathbf{F}_{i-m_{i}+1,3} & =\Delta t^{2} \mathbf{A}_{i+1} \mathbf{F}_{i-m_{i}+1,2}+\Delta t \mathbf{B}_{i+1} .
\end{aligned}
$$

By substituting (19)-(21) into (18), we can obtain the following iterative formula:

$$
\mathbf{x}_{i+1}=\mathbf{F}_{i} \mathbf{x}_{i}+\mathbf{F}_{i-m_{i}} \mathbf{x}_{i-m_{i}}+\mathbf{F}_{i-m_{i}+1} \mathbf{x}_{i-m_{i}+1},
$$

where the coefficients can be expressed as

$$
\begin{aligned}
\mathbf{F}_{i} & =\mathbf{I}+\frac{1}{6} \mathbf{A}_{i}+\frac{1}{3} \mathbf{F}_{i, 1}+\frac{1}{3} \mathbf{F}_{i, 2}+\frac{1}{6} \mathbf{F}_{i, 3}, \\
\mathbf{F}_{i-m_{i}} & =\frac{1}{6} \mathbf{B}_{i}+\frac{1}{3} \mathbf{F}_{i-m_{i}, 1}+\frac{1}{3} \mathbf{F}_{i-m_{i}, 2}+\frac{1}{6} \mathbf{F}_{i-m_{i}, 3}, \\
\mathbf{F}_{i-m_{i}+1} & =\frac{1}{3} \mathbf{F}_{i-m_{i}+1,1}+\frac{1}{3} \mathbf{F}_{i-m_{i}+1,2}+\frac{1}{6} \mathbf{F}_{i-m_{i}+1,3},
\end{aligned}
$$

where $\mathbf{I}$ is an $n \times n$ identity matrix and $n$ is the dimension of vector $\mathrm{x}(t)$.

To obtain the transition matrix, we first define a new $n \times(M+1)$ dimensional vector $z_{i}$ as

$$
\mathrm{z}_{i}=\operatorname{col}\left(\mathrm{x}_{i}, \mathrm{x}_{i-1}, \ldots, \mathrm{x}_{i-M+1}, \mathrm{x}_{i-M}\right) .
$$

Finally, the resulting discrete map is expressed as

$$
\mathrm{z}_{i+1}=\mathrm{D}_{i} \mathrm{z}_{i}
$$

where the coefficient matrix $D_{i}$ can be constructed as a $(2 M+4)$-dimensional matrix:

$$
\mathrm{D}_{i}=\left[\begin{array}{ccccccc}
F_{i} & 0 & 0 & \ldots & 0 & \mathrm{~F}_{i-m_{i}+1} & \mathrm{R}_{i-m_{i}} \\
\mathrm{I} & 0 & 0 & \ldots & 0 & 0 & 0 \\
0 & \mathrm{I} & 0 & \ldots & 0 & 0 & 0 \\
\vdots & \vdots & \vdots & \ddots & \vdots & \vdots & \vdots \\
0 & 0 & 0 & \ldots & 0 & 0 & 0 \\
0 & 0 & 0 & \ldots & \mathrm{I} & 0 & 0 \\
0 & 0 & 0 & \ldots & 0 & \mathrm{I} & 0
\end{array}\right] .
$$

Therefore, the approximate Floquet transition matrix can be described as

$$
\Phi=\mathrm{D}_{k-1} \mathrm{D}_{k-2} \cdots \mathrm{D}_{1} \mathrm{D}_{0} .
$$

Then, the stability of the system can be calculated based on the Floquet theory. If the moduli of all eigenvalues of the transition matrix $\Phi$ are less than unity, then the system is stable; otherwise, it is unstable.

\section{Simulation and Discussion}

In this section, an experimental example [3] is used to verify the accuracy and efficiency of RKCDM. The modal parameters of the milling systems are tool diameter $D=12.7$, number of teeth $N=2$, tangential cutting force coefficient $K_{t}=6 \times 10^{8} \mathrm{~N} / \mathrm{m}^{2}$, radial cutting force coefficient $K_{n}=$ $2 \times 10^{8} \mathrm{~N} / \mathrm{m}^{2}$, natural frequency $\omega_{\mathrm{nx}}=\omega_{\mathrm{ny}}=922.0 \mathrm{~Hz}$, modal damping ratio $\zeta_{x}=\zeta_{y}=0.011$, and modal mass $m_{\mathrm{tx}}=m_{\mathrm{ty}}=0.03993 \mathrm{~kg}$. All calculations are performed in MATLAB R2014a on a desktop computer (Intel Core (TM) i7-6700K central processing unit, $4.0 \mathrm{GHz}, 16 \mathrm{~GB})$. We first discuss the convergence of RKCDM and SDM. 


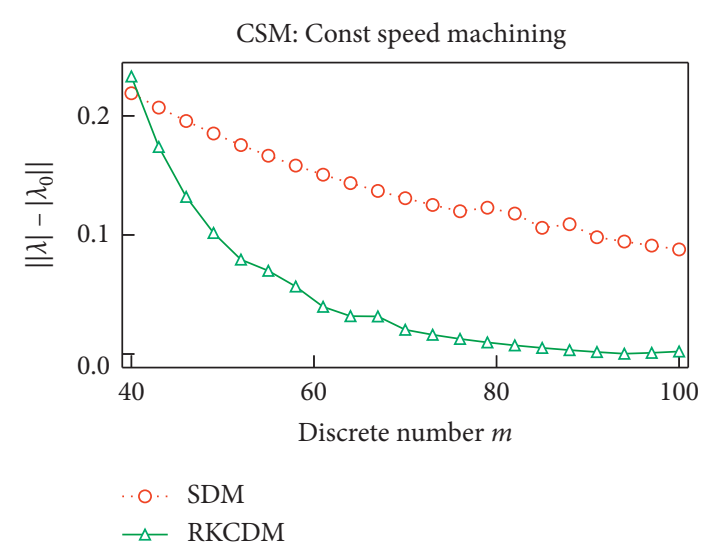

(a)

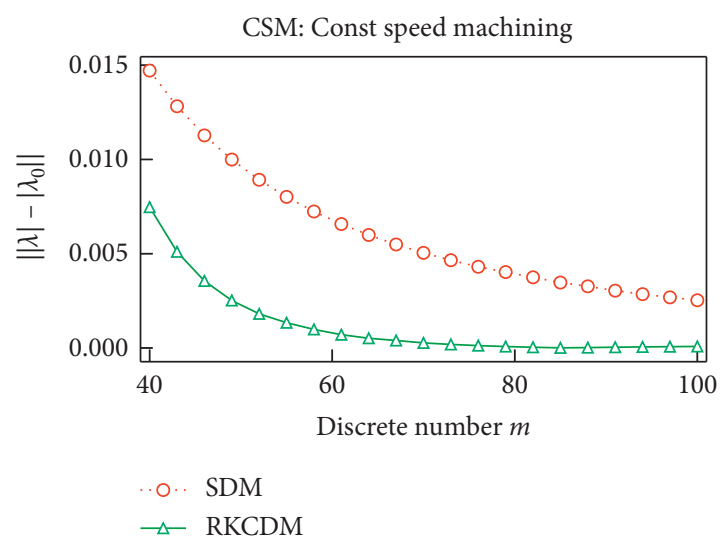

(c)

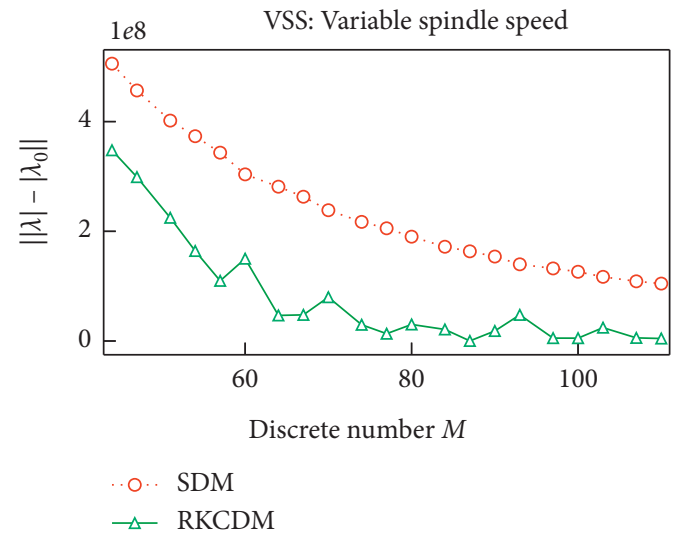

(e)

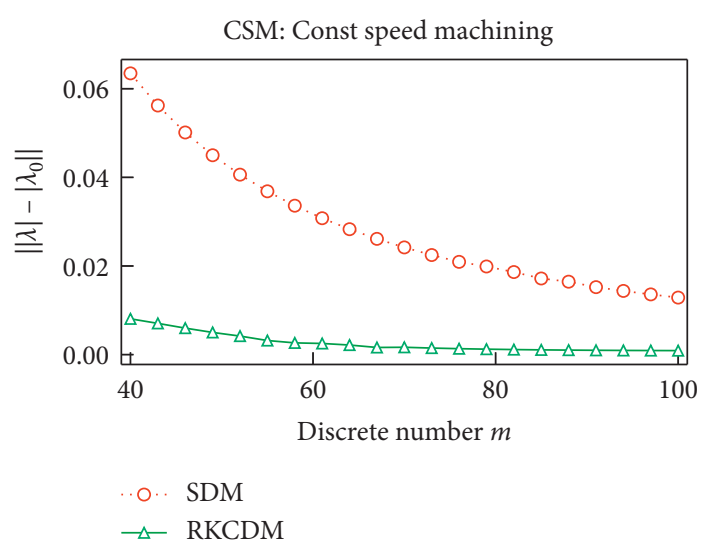

(b)

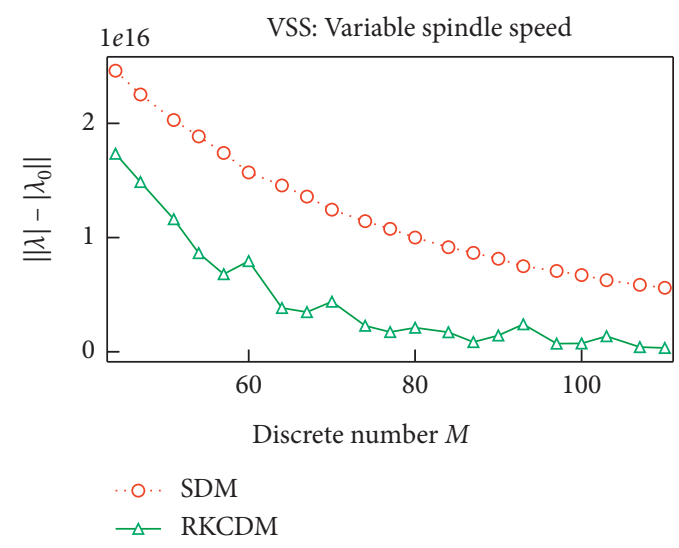

(d)

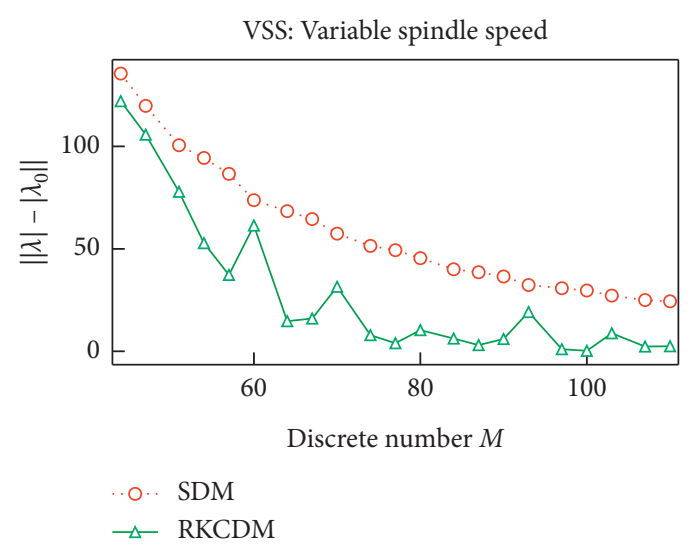

(f)

FIGURE 4: Convergence rate comparisons of SDM and RKCDM for constant-speed machining and variable-spindle-speed machining. The spindle speed (nominal spindle speed for VSS milling) is 7,000 rpm: (a) $w=1 \mathrm{~mm}$, (b) $w=0.5 \mathrm{~mm}$, (c) $w=0.1 \mathrm{~mm}$, (d) $w=1 \mathrm{~mm}$, (e) $w=0.5 \mathrm{~mm}$, and (f) $w=0.2 \mathrm{~mm}$. RVA $=\mathrm{RVF}=0.3$ for (d), (e), and (f).

4.1. Convergence and Computation Efficiency. The convergence rates of RKCDM and SDM with different conditions are compared in Figure 4. The discrete number of time delays $\tau$ ( $\tau(t)$ for VSS milling) is set as $m$ for constant-speed milling and $M$ for VSS milling and is determined according to (17), and the maximal module of the eigenvalues of the transition matrix $\Phi$ is set as $\lambda$. To compare the convergence of RKCDM and SDM, the value of $\lambda$ under condition of $m=500$ is written as $\lambda_{0}$ as the exact value to assess its convergence rate. When $m$ increases, the approximation error of RKCDM is lower than SDM. The same phenomenon can be obtained for VSS milling with the increase of $M$. This means that RKCDM converges faster than SDM with the increase of the discrete number of time delays, because for SDM, the discretization error is $O(h)^{2}$ [36]. However, discretization error for RKCDM is $O(h)^{5}$. Niu et al. [32] used two-point barycentric Lagrange points to approximate the middle points, so the remainder of the 

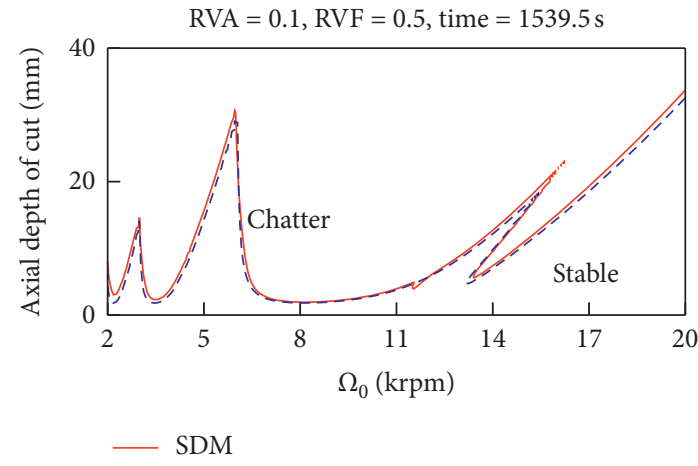

-- CSS

(a)

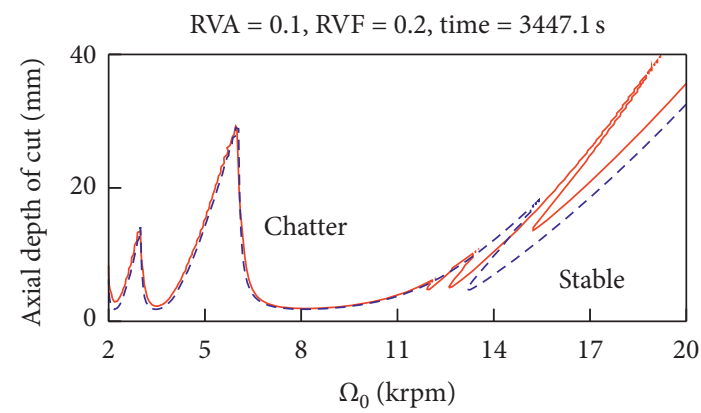

_ SDM

. - . CSS

(c)

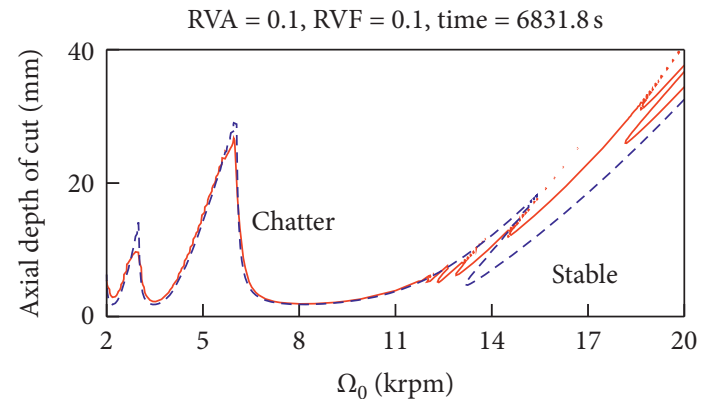

— SDM

-.. CSS

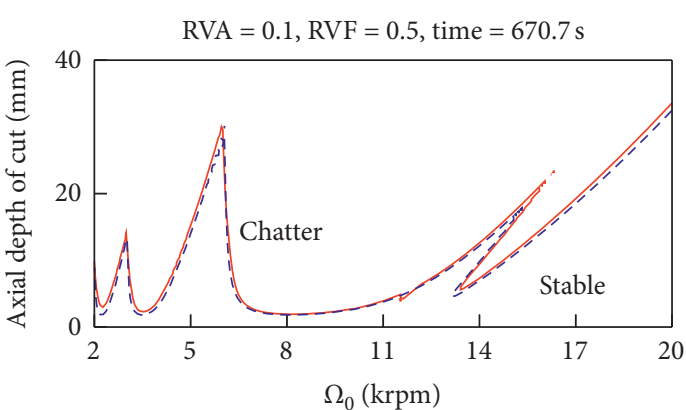

- RKCDM

-.. CSS

(b)

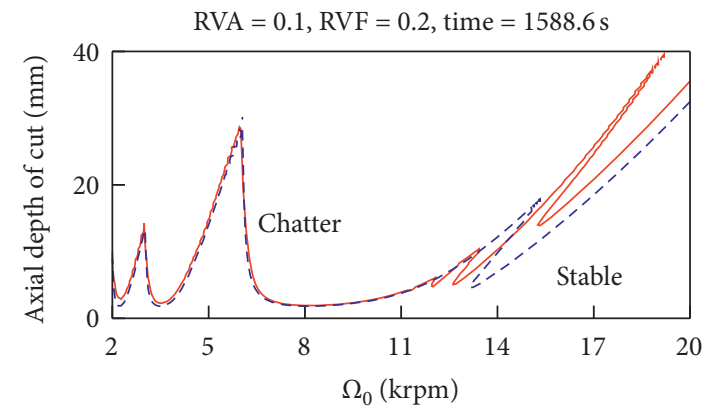

- RKCDM

. - - CSS

(d)

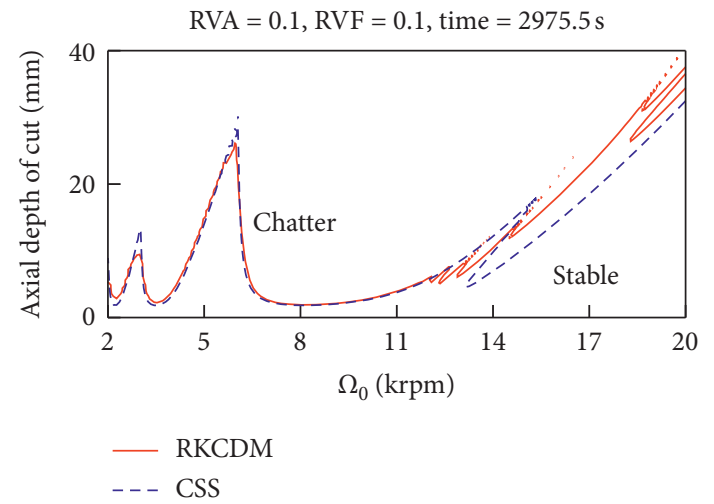

(f)

(e)

FIGURE 5: Stability charts of milling with spindle-speed modulation obtained over $200 \times 100$ size grid of spindle speed and ADOC, aD $=0.5$, $K=80 /$ RVF, experimental data from [34].

two-point interpolation is $O(h)^{2}$. To increase the accuracy of RKCDM, $2 k$ discrete points are taken in one toothpassing interval to calculate cutting-force coefficients $h_{\mathrm{xx}}(t), h_{\mathrm{xy}}(t), h_{\mathrm{yx}}(t)$, and $h_{\mathrm{yy}}(t)$ for the following examples. So, at time interval $\left[t_{i}, t_{i+1}\right], \mathbf{A}_{i}, \mathbf{A}_{i+1}, \mathbf{B}_{i}$, and $\mathbf{B}_{i+1}$, can be calculated using even points, and $\mathbf{A}_{i+0.5}$ and $\mathbf{B}_{i+0.5}$ are calculated using the left points. The benefit of this is that the accuracy of RKCDM is the same with the classical fourthorder Runge-Kutta method. To improve the prediction accuracy, the discrete number $M$ of time-varying delays $\tau(t)$ is always greater than 80 , and according to Figure 4, RKCDM can have the minimum approximation error.

To compare the computational efficiency, we refer to Figure 5, which shows the time consumption using RKCDM and SDM. For VSS milling, the consumption time to achieve stability increases as RVA decreases. For small RVA, more time can be saved with RKCDM than with SDM. Hence, we conclude that the time consumption increases with the number of discrete time delays. The results of Figure 5 indicate that $54 \%$ of time consumption can be saved using RKCDM instead of SDM. 


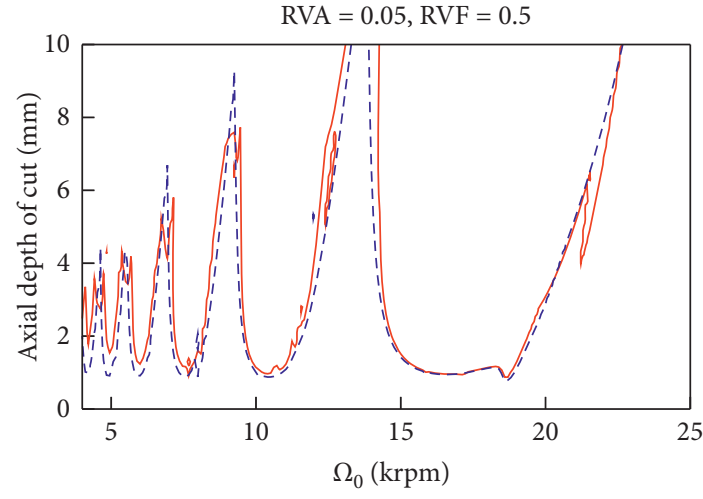

— VSS

- - CSS

(a)

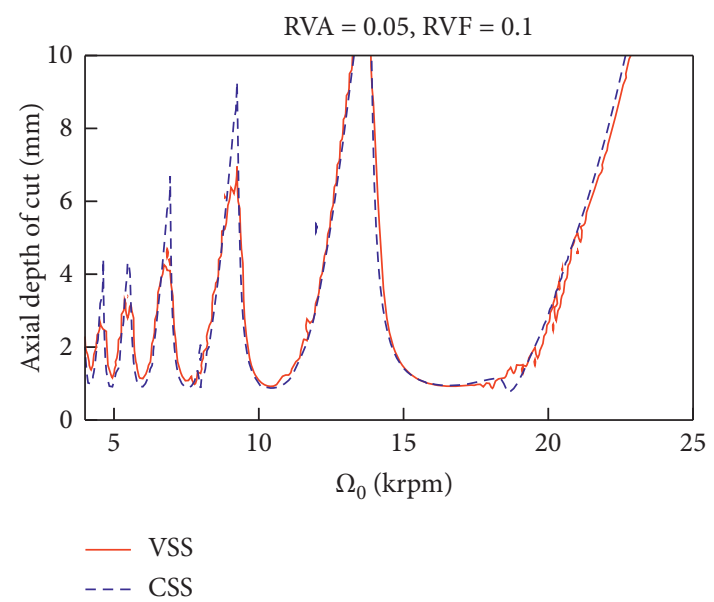

(b)

FIgURE 6: Stability lobe diagram of CSS and VSS milling: $\mathrm{aD}=0.1$.

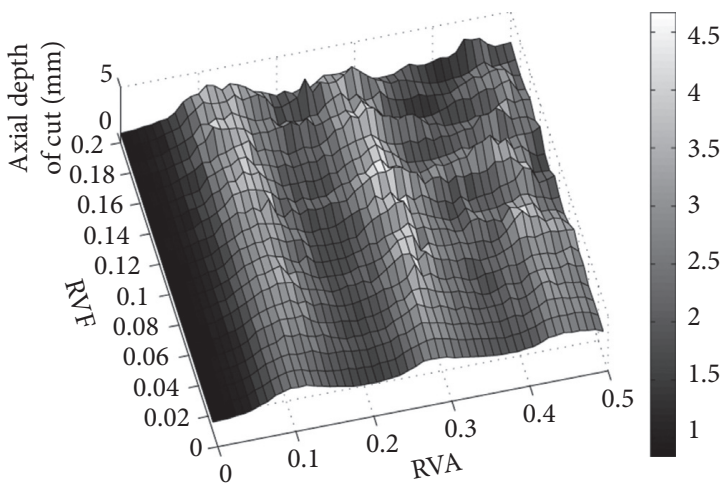

(a)

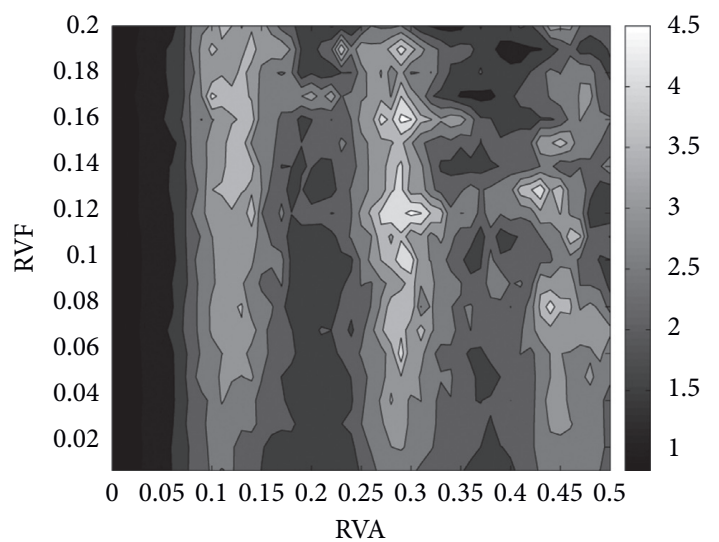

(b)

Figure 7: Nominal spindle speed 4,900 rpm: (a) map of limit ADOC for sinusoidal modulation VSS milling; (b) contour plot of limit ADOC for sinusoidal modulation VSS milling.

4.2. Analysis of Effect of Modulation Parameters on Stability of VSS Milling. Figure 6 shows the SLD of VSS and CSS milling. Using VSS, the stability boundary obviously increases, especially at low spindle speeds. Although the depth of cut increases using VSS, the modulation also has an important effect on the stability. Taking Figure 6(a) as an example, the depth of cut around nominal spindle speed $22,000 \mathrm{rpm}$ decreases abruptly. To comprehensively explore the effects of modulation parameters on the stability of milling, the relationships among depth of cut, RVA, and RVF are shown in the 3D plots of Figures 7 and 8. According to Figure 6(a), the axial depth of cut has a minimum value of $1 \mathrm{~mm}$ for CSS milling.

After VSS is used, the ADOC at these two nominal spindle speeds increases significantly, as illustrated in Figures 7 and 8 . Figure 7 shows that the ADOC changes with the variation of sinusoidal modulation parameters RVA and RVF around a nominal spindle speed of $4,900 \mathrm{rpm}$. Obviously, when VSS is used, the lowest point is higher than that of CSS milling. From Figure 7, we know that the ADOC is more sensitive to RVA than to RVF; so, for the machinist, the selection of modulation parameters is more interesting than that of spindle speed. Taking a nominal spindle speed of $4,900 \mathrm{rpm}$ as an example, with modulation parameters $\mathrm{RVA}=0.10$ and $\mathrm{RVF}=0.17, \mathrm{ADOC}$ will increase to $4.16 \mathrm{~mm}$. The contour plot in Figure 7(b) shows that not only at the vicinity of point $(0.10,0.17)$ but also all stable ADOC limit in these two figures (Figures 7 and 8) are greater than $1 \mathrm{~mm}$ too. So, VSS can always increase the ADOC around the nominal spindle speed of $4,900 \mathrm{rpm}$. When RVA is in the range [0.26, 0.33 ], the ADOC can still achieve a local maximum. Because the selection of RVA is constrained by the driving power of the spindle speed, a low RVA is more adaptable for VSS milling.

The results at a nominal spindle speed of $22,000 \mathrm{rpm}$ differ from those at $4,900 \mathrm{rpm}$. ADOC drops abruptly around a nominal spindle speed of 22,000 rpm. To explore the effects of the VSS technique on the stability of milling, we 


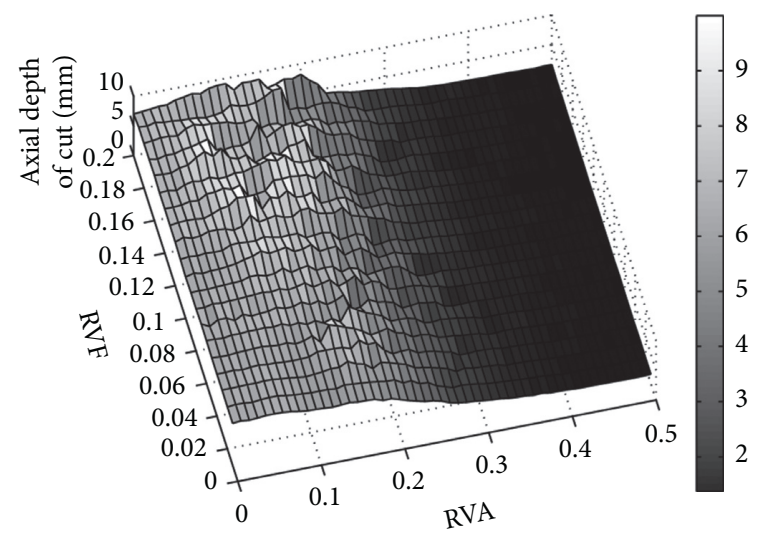

(a)

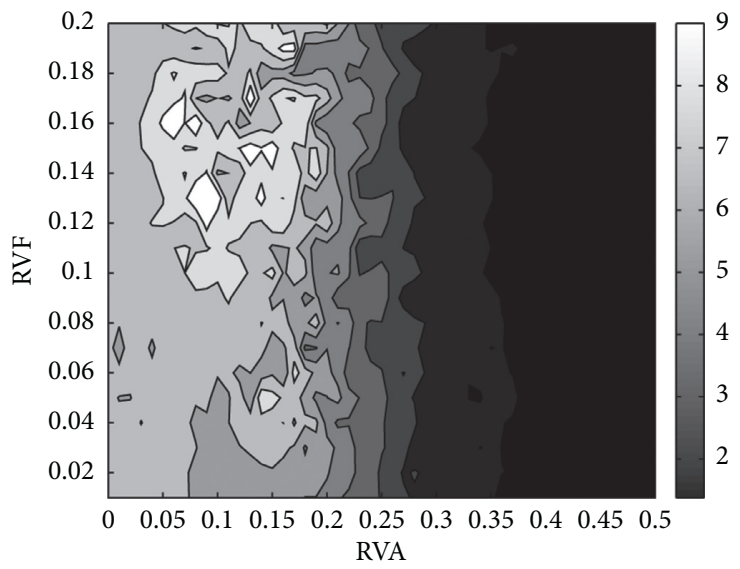

(b)

FIgURE 8: Nominal spindle speed 22,000 rpm: (a) map of limit ADOC for sinusoidal modulation VSS milling; (b) contour plot of limit ADOC for sinusoidal modulation VSS milling.

show a three-dimensional stability diagram at a nominal spindle speed of 22,000 rpm in Figure 8. With VSS, ADOC is always higher than CSS in Figure 7. However, this is different at a nominal spindle speed of $22,000 \mathrm{rpm}$, where ADOC does not always increase with VSS milling over CSS milling. Figure 6 shows that ADOC is $7.83 \mathrm{~mm}$ without VSS. In Figure 8, ADOC is always lower than $7.83 \mathrm{~mm}$ with RVA in the range $[0.2,0.3]$. So, it is important to choose modulation parameters appropriately to improve stability with the VSS technique. In Figure 8, ADOC increases to a local summit with the variation of RVA and RVF, such as around $\mathrm{RVA}=0.06$ and $\mathrm{RVF}=0.16, \mathrm{RVA}=0.08$ and $\mathrm{RVF}=0.13$, and $\mathrm{RVA}=0.14$ and $\mathrm{RVF}=0.15$. Taking $\mathrm{RVA}=0.06$ and $\mathrm{RVF}=0.16$ as an example, the ADOC can reach $9.99 \mathrm{~mm}$ in this area. So, ADOC can still be increased with appropriate selection of modulation parameters.

\section{Conclusion}

A new algorithm for stability predictions of VSS milling was developed, and the following conclusions were drawn:

(1) The governing DDE of the time-periodic milling process including the regenerative effect is described by a set of algebraic equations, and the stability of the system is predicted by the Floquet theory.

(2) Comparisons of convergence rate and computational efficiency were conducted. RKCDM shows a higher convergence rate than SDM, so RKCDM is more accurate.

(3) Simulation results indicate that RKCDM can be used for parameter selecting in VSS milling. The maps and contour plots of ADOC produced from RKCDM show the stability of VSS milling with sinusoidal modulation changes with RVA and RVF, and the stability is more sensitive to variation in RVA. The maps and contour plots of ADOC can be used to select the optimal modulation parameters for VSS milling.
Finally, although the optimal parameters of the VSS technique have been selected through the method proposed here, it is obvious that the parameters should be further tested, and this will be our future work.

\section{Data Availability}

The data used to support the findings of this study are included within the article.

\section{Conflicts of Interest}

The authors declare no conflicts of interest.

\section{Acknowledgments}

This work was financially supported by the Science and Technology Commission of Shanghai Municipality (17DZ2283300).

\section{References}

[1] Y. Altintas and E. Budak, "Analytical prediction of stability lobes in milling," CIRP Annals-Manufacturing Technology, vol. 44, pp. 357-362, 1995.

[2] Y. Ding, L. M. Zhu, X. J. Zhang et al., "Numerical integration method for prediction of milling stability," Journal of Manufacturing Science and Engineering, vol. 133, Article ID 031005, 2011.

[3] P. V. Bayly, B. P. Mann, T. L. Schmitz et al., "Effects of radial immersion and cutting direction on chatter instability in endmilling," in Proceedings of the ASME International Mechanical Engineering Congress and Exposition, pp. 351-363, Dallas, TX, USA, November 1996.

[4] T. Insperger and G. Stepan, "Stability analysis of turning with periodic spindle speed modulation via semidiscretization," Journal of Vibration and Control, vol. 10, no. 12, pp. 18351855, 2004.

[5] T. Insperger, G. Stépán, and J. Turi, "On the higher-order semi-discretizations for periodic delayed systems," Journal of Sound and Vibration, vol. 313, no. 1-2, pp. 334-341, 2008. 
[6] X. Dong, W. Zhang, and S. Deng, "The reconstruction of a semi-discretization method for milling stability prediction based on Shannon standard orthogonal basis," The International Journal of Advanced Manufacturing Technology, vol. 85, no. 5-8, pp. 1501-1511, 2016.

[7] X. Dong and W. Zhang, "Stability analysis in milling of the thin walled part considering multiple variables of manufacturing systems," The International Journal of Advanced Manufacturing Technology, vol. 89, no. 1-4, pp. 515527, 2017.

[8] S. Yi, P. W. Nelson, and A. G. Ulsoy, "Delay differential equations via the matrix Lambert $\mathrm{W}$ function and bifurcation analysis: application to machine tool chatter," Mathematical Biosciences and Engineering: MBE, vol. 4, no. 2, pp. 355-368, 2007.

[9] Y. Ding, X. J. Zhang, and H. Ding, "A legendre polynomials based method for stability analysis of milling processes," Journal of Vibration and Acoustics, vol. 137, Article ID 024504, 2015.

[10] C. Qin, J. Tao, and C. Liu, "Stability analysis for milling operations using an Adams-Simpson-based method," The International Journal of Advanced Manufacturing Technology, vol. 92, no. 1-4, pp. 969-979, 2017.

[11] Z. Li, Z. Yang, Y. Peng, F. Zhu, and X. Ming, "Prediction of chatter stability for milling process using Runge-Kutta-based complete discretization method," The International Journal of Advanced Manufacturing Technology, vol. 86, no. 1-4, pp. 943-952, 2016.

[12] E. A. Butcher, P. Nindujarla, and E. Bueler, "Stability of upand down-milling using Chebyshev collocation method," in Proceedings of ASME 2005 International Design Engineering Technical Conferences and Computers and Information in Engineering Conference IDETC/CIE, Oakland, CA, USA, September 2005.

[13] J. Slavicek, "The effect of irregular tooth pitch on stability of milling," in Proceedings of the 6th MTDR Conference, pp. 15-22, Pergamon Press, London, UK, September 1965.

[14] Y. Altintas, S. Engin, and E. Budak, "Analytical stability prediction and design of variable pitch cutters," Journal of Manufacturing Science and Engineering, vol. 121, pp. 173-178, 1999.

[15] N. Olgac and R. Sipahi, "Dynamics and stability of variablepitch milling," Journal of Vibration and Control, vol. 13, no. 7, pp. 1031-1043, 2007.

[16] G. Jin, Q. Zhang, S. Hao et al., "Stability prediction of milling process with variable pitch cutter," Mathematical Problems in Engineering, vol. 2013, Article ID 932013, 11 pages, 2013.

[17] N. D. Sims, B. Mann, and S. Huyanan, "Analytical prediction of chatter stability for variable pitch and variable helix milling tools," Journal of Sound and Vibration, vol. 317, no. 3-5, pp. 664-686, 2008.

[18] T. Stoferle and H. Grab, "Vermeiden von Ratterschwingungen durch periodische Drehzahlanderung," Werkstatt und Betrieb, vol. 105, p. 727, 1972.

[19] T. Takemura, T. Kitamura, and T. Hoshi, "Active suppression of chatter by programmed variation of spindle speed," CIRP Annals, vol. 23, pp. 121-122, 1974.

[20] Y. Altintas and P. K. Chan, "In-process detection and suppression of chatter in milling," International Journal of Machine Tools and Manufacture, vol. 32, no. 3, pp. 329-347, 1992.

[21] T.-C. Tsao, M. W. McCarthy, and S. G. Kapoor, "A new approach to stability analysis of variable speed machining systems," International Journal of Machine Tools and Manufacture, vol. 33, no. 6, pp. 791-808, 1993.
[22] X. H. Long and B. Balachandran, "Stability of up-milling and down-milling operations with variable spindle speed," Journal of Vibration and Control, vol. 16, pp. 1151-1168, 2010.

[23] M. Zatarain, I. Bediaga, J. Muñoa, and R. Lizarralde, "Stability of milling processes with continuous spindle speed variation: analysis in the frequency and time domains, and experimental correlation," CIRP Annals, vol. 57, no. 1, pp. 379-384, 2008.

[24] S. Seguy, T. Insperger, L. Arnaud, G. Dessein, and G. Peigné, "On the stability of high-speed milling with spindle speed variation," The International Journal of Advanced Manufacturing Technology, vol. 48, no. 9-12, pp. 883-895, 2010.

[25] S. Seguy, T. Insperger, L. Arnaud, G. Dessein, and G. Peigné, "Suppression of period doubling chatter in high-speed milling by spindle speed variation," Machining Science and Technology, vol. 15, no. 2, pp. 153-171, 2011.

[26] Q. Xie and Q. Zhang, "Stability predictions of milling with variable spindle speed using an improved semi-discretization method," Mathematics and Computers in Simulation, vol. 85, pp. 78-89, 2012.

[27] Q. Xie, Q. Zhang, W. Wang, G. Jin, and J. Han, "Stability analysis for variable spindle speed milling with helix angle using an improved semi-discretization method," Science China Technological Sciences, vol. 56, no. 3, pp. 648-655, 2013.

[28] Y. Ding, J. B. Niu, L. M. Zhu et al., "Numerical integration method for stability analysis of milling with variable spindle speeds," ASME Journal of Vibration and Acoustics, vol. 138, Article ID 011010, 2015.

[29] J. B. Niu, Y. Ding, L. M. Zhu et al., "Stability analysis of milling processes with periodic spindle speed variation via the variable-step numerical integration method," Journal of Manufacturing Science and Engineering, vol. 138, Article ID 114501, 2016.

[30] G. Totis, P. Albertelli, M. Sortino, and M. Monno, "Efficient evaluation of process stability in milling with spindle speed variation by using the Chebyshev collocation method," Journal of Sound and Vibration, vol. 333, no. 3, pp. 646-668, 2014.

[31] G. Jin, H. Qi, Z. Li, and J. Han, "Dynamic modeling and stability analysis for the combined milling system with variable pitch cutter and spindle speed variation," Communications in Nonlinear Science and Numerical Simulation, vol. 63, pp. 38-56, 2018.

[32] J. Niu, Y. Ding, L. Zhu, and H. Ding, "Runge-Kutta methods for a semi-analytical prediction of milling stability," Nonlinear Dynamics, vol. 76, no. 1, pp. 289-304, 2014.

[33] M. Li, G. Zhang, and Y. Huang, "Complete discretization scheme for milling stability prediction," Nonlinear Dynamics, vol. 71, no. 1-2, pp. 187-199, 2013.

[34] T. Insperger and G. Stepan, Semi-Discretization for TimeDelay Systems: Stability and Engineering Applications, Springer, New York, NY, USA, 2011.

[35] J. Kiusalaas, Numerical Methods in Engineering with python3, Cambridge University Press, Cambridge, UK, 2013.

[36] T. Insperger, "Full-discretization and semi-discretization for milling stability prediction: some comments," International Journal of Machine Tools and Manufacture, vol. 50, no. 7, pp. 658-662, 2010. 